\title{
Contribución de los establecimientos de salud en la educación en salud en Honduras
}

Contribution of health facilities in health education in Honduras

El sistema de salud en Honduras contribuye de manera importante a la formación médica, facilitando espacios, talento humano y pacientes. La evaluación constante de los programas de formación médica ha demostrado el impacto que la formación médica tiene en los médicos y los pacientes, además nos muestra los retos y posibles soluciones. El ambiente donde este se construye debe tener ciertas características para lograr un resultado óptimo. ${ }^{1}$

En Honduras se crean las especialidades médicas en la década de los años setenta como una iniciativa de especialistas docentes asistenciales del Hospital Materno Infantil de acuerdo al convenio entre la Universidad Nacional Autónoma de Honduras (UNAH) y el Ministerio de Salud Pública (MSP) de la época, y como aval el Colegio Médico de Honduras (CMH). Es así como el $1^{\circ}$ de marzo de 1975 inician las residencias médicas en la especialidad de Pediatría y la especialidad de Ginecología y Obstetricia. En 1978, con el inicio de actividades médicas del Bloque Médico Quirúrgico, pasa a ser el Hospital Escuela. Desde 1975 al 2019 se han implementado 24 especialidades y subespecialidades, contribuyendo de esta forma a ofrecer diferentes opciones de formación médica profesional. ${ }^{2}$

La República de Honduras cuenta con establecimientos de salud en todos sus 18 departamentos, estando estos categorizados en establecimientos del primero y segundo nivel de atención, de acuerdo a su complejidad. ${ }^{3}$ El primer nivel, eminentemente ambulatorio, tiene tres niveles y el segundo nivel, hospitalario, tiene cuatro niveles, desde hospital con especialidades básicas hasta el hospital universitario. La clasificación por categorías determina el tipo de establecimiento necesario para atender las demandas de salud de la población. Esto constituye uno de los aspectos importantes de la organización de la oferta, porque permite organizar y consolidar redes asistenciales articulados por niveles de complejidad, un sistema de referencia y respuesta, que permita un mecanismo de coordinación para asegurar una atención oportuna integral y completa del paciente con el objetivo de dar seguimiento al manejo y evolución del mismo. ${ }^{3} \mathrm{El}$ sistema de salud, además de su red pública de establecimientos, cuenta con la red del Instituto Hondureño de Seguridad Social (IHSS) y la red de establecimientos de salud privada. En cada uno de los establecimientos los profesionales de la medicina aprenden con cada caso tratado y comparten experiencias entre colegas las cuales contribuyen a su crecimiento profesional.

La medicina se aprende al pie del enfermo, con buenos maestros y una buena metodología de aprendizaje. A través del tiempo, la medicina ha cambiado revolucionariamente y cada vez es más compleja la naturaleza del conocimiento médico. Sin lugar a dudas, el hospital desempeña un papel central para la formación y aprendizaje del médico. ${ }^{4}$ La contribución de los establecimientos de salud públicos, del IHSS y establecimientos del sector privado a través de los años, ha representado un factor clave en el aprendizaje y avance de la medicina. A pesar de contar con un sistema de salud público actualmente al año 2021 colapsado y superado por la pandemia de COVID-19, centros hospitalarios con infraestructuras deficientes, con carencias de insumos y medicamentos, con avances tecnológicos limitados, hay profesionales de la medicina junto con otro personal de salud comprometido en investigar, compartir conocimientos y avanzar en la búsqueda de la excelencia en la atención para beneficiar a quien más lo necesita, quien es la razón para avanzar: el paciente. Aún hay muchos retos y desafíos que vencer. El más importante, no desmayar y seguir adelante a pesar de la impunidad y la corrupción del sistema.

Rosa María Duarte

Doctora en Medicina y Cirugía, Magíster en Salud Pública

Consejo Editorial

Revista Médica Hondureña

\section{REFERENCIAS}

1. Solano J. Percepción de factores modificables que afectan la educación médica en Honduras: Encuesta 2019. Rev Méd Hondur. [Internet]. 2019[consultado 23 marzo 2021]; 87 (2): 55-62. Disponible en: http:// www.bvs.hn/RMH/pdf/2019/pdf/Vol87-2-2019-4.pdf

2. Universidad Nacional Autónoma de Honduras. Facultad de Ciencias Médicas, Postgrados. [Internet]. Tegucigalpa: UNAH, FCM; 2020. [consultado 15 abril 2021]. Disponible en: http://www.bvs.hn/php/level.php?lang=e s\&component $=35 \&$ item $=103$

3. Secretaria de Salud (HN). Modelo Nacional de Salud. Tegucigalpa: Secretaria de Salud; 2013. [consultado 15 abril 2021]. Disponible en: https:// bit.ly/2QmAPMn

4. Martínez-Hernández J. Integrar la enseñanza pregrado en el hospital. Educ. Med. 2008;11(supl 1):S33-S36. 